\title{
Zum Nachwuchsmangel in Unfallchirurgie und Orthopädie
}

\author{
On the Lack of Young Physicians in Trauma Surgery and Orthopaedics
}

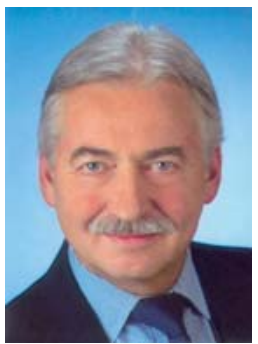

K. Weise

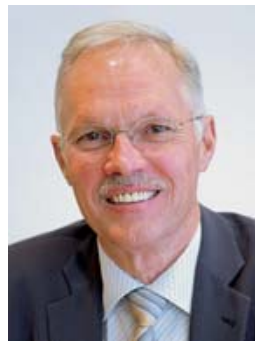

F. U. Niethard

Bibliografie

DOI $10.1055 / \mathrm{s}-0029-1240871$ Z Orthop Unfall 2010; 148: 1718 ๔ Georg Thieme Verlag KG Stuttgart · New York . ISSN 1864-6697

\section{Korrespondenzadressen} Prof. Dr. med. Kuno Weise BG-Unfallklinik

Schnarrenbergstraße 95 72076 Tübingen

Tel.: 0 07071/606-1001

Fax: 07071/606-1002

weise@bgu-tuebingen.de

Prof. Dr. med. Fritz U. Niethard Orthopädische Klinik Universitätsklinik der RWTH Aachen

Pauwelsstraße 30

52074 Aachen

Tel.: 0241/8 08-94 10

Fax: 0241/808-2453

funiethard@

orthopaedie-aachen.de
„Neben allgemeiner Kritik an der Einkommenssituation, dem Ausmaß der zu bewältigenden nichtärztlichen Aufgaben sowie einer zunehmenden Fremdbestimmung und Bürokratisierung gibt es in der Chirurgie vor allem Kritik über eine mangelnde Strukturierung der Weiterbildung, über lange Arbeitszeiten mit kaum möglicher Vereinbarkeit von Berufs- und Familienleben, ein immer noch stark geprägtes Hierarchieverständnis und Mängel in der fachlichen Betreuung."

(Prof. Dr. H. Bauer, Generalsekretär der Deutschen Gesellschaft für Chirurgie) [3]

Das Editorial in Heft 1/2009 der ZfOU beschäftigte sich mit den Wünschen und Vorstellungen, die das Junge Forum der DGOU in verschiedenen Verlautbarungen und Publikationen thematisierte [8]. Ein Jahr später sind die Sorgen um den chirurgischen Nachwuchs sicherlich nicht kleiner geworden, wohl aber wurden die Ursachen für die noch immer unzureichende Zahl nachrückender Chirurginnen und Chirurgen näher beleuchtet und man erkennt zunehmende Aktivitäten der Fachgesellschaften, um dieses ernsthaften Problems Herr zu werden [4].

Herr Dr. Albrecht, als Oberarzt in nicht selbstständiger Stellung einer chirurgischen Universitätsklinik (Eberhard Karls Universität, Tübingen) im Präsidium der Deutschen Gesellschaft für Chirurgie, hat in den Mitteilungen der Deutschen Gesellschaft für Chirurgie 1/2010 Gründe für den Nachwuchsmangel in unseren Fachgebieten aufgezeigt und sich darüber Gedanken gemacht, wie man die Attraktivität der chirurgischen Fächer für Absolventen des Medizinstudiums erhöhen kann [1]. Zwar ist laut H. Bauer die Zahl der Chirurgen während der vergangenen 10 Jahre im Vergleich zu allen anderen Arztgruppen überproportional gestiegen, gleichwohl könnten bundesweit Assistentenstellen in chirurgischen Abteilungen immer schwerer besetzt werden [3]. Diese Meinung teilt auch Albrecht, indem er in seinen Ausführungen auf den Umstand hinweist, dass die Kliniken vor Jahren ihre neuen Mitarbeiter aus einem umfangreichen Bewerberpool rekrutieren konnten, wohingegen nunmehr eine Umkehrung des Verhältnisses Bewerber/Stellenausschreibung eingetreten sei, indem erstere sich ihren zukünftigen Arbeitsplatz auswählen könnten. Die Gründe hierfür sind laut Albrecht vielfältiger Natur, es gebe aber 3 wesentliche Faktoren, nämlich den hohen Frauenanteil der Studierenden (sog. Feminisierung der Medizin), den Wertewandel in der neuen Generation (Stichwort: „work-life balance“) und den Attraktivitätsverlust chirurgischer Fächer während der studentischen Ausbildung. Seine weiteren Ausführungen zu diesen 3 zentralen Ursachen für den Nachwuchsmangel in der Chirurgie umfassen neben einer Bestandsaufnahme im Hinblick auf den Status quo eine Reihe von Vorschlägen, die zusammengefasst nachstehende Initiativen beinhalten [1]:

1. Wichtigster Kritikpunkt weiblicher Absolventen des Medizinstudiums bezüglich des Berufsziels „Chirurgie“ ist die Unvereinbarkeit von Familienplanung einerseits und den Rahmenbedingungen der chirurgischen Weiterbildung (z.B. ungünstige Arbeitszeiten) andererseits. Zudem fühlen sich laut einer Umfrage 65\% weiblicher Studierender durch herablassendes oder übertrieben freundliches Verhalten männlicher Dozenten bzw. Kollegen diskriminiert. Frau Dr. Bühren bescheinigt auf der Basis dieser Umfrage des Deutschen Ärztinnenbunds der Chirurgie familienfeindliche Strukturen. Demzufolge propagiert Albrecht in seinen zusammenfassenden Vorschlägen bezüglich geeigneter Maßnahmen zur Beseitigung des Bewerbermangels die Förderung familienfreundlicher Rahmenbedingungen, wie beispielsweise Angebote zur Kinderbetreuung, was zum einen die Zahl weiblicher Mitarbeiter in den chirurgischen Fächern erhöhen könnte und nach der Schwangerschaft und Elternzeit eine erhöhte Rückkehr an den früheren Arbeitsplatz zur Folge hätte. Gleichzeitig sollte man mit der überkommenen Vorstellung aufräumen, so Albrecht, dass chirurgische Fächer nichts für Frauen seien, weil die Tätigkeit körperlich wie psychisch eine zu hohe Belastung darstellte. Frau PD Dr. Seifert thematisiert hierzu in einem Aufsatz über den Nachwuchsmangel in der Unfallchirurgie, dass in den chirurgischen Fächern nach wie vor eine den militärischen wie männlich geprägten Traditionen entsprechende Hierarchie bestünde, gleichzeitig werde die besondere körperliche und psychische Arbeitsbelastung von der jetzigen Generation der Studierenden als unangemessen empfunden. Außerdem gebe es aufgrund der Tatsache, dass Unfallchirurginnen in Chef- oder Oberarztpositionen eine Rarität seien, für angehende Chirurginnen zu wenig Vorbilder [7]. Ein Bericht über den Auftakt der Kampagne „nur Mut“ des BDC weist darauf 
hin, dass die Chirurgie in ihrem Handwerk keineswegs frauenfeindlich sei, indem man nicht immer Muskelkraft benötige und Frauen bei chirurgischer Tätigkeit ihren männlichen Kollegen keineswegs nachstünden [2].

Zusammenfassend kann demnach eine Erhöhung der Zahl weiblicher Chirurgen nur dadurch erreicht werden, dass man die Rahmenbedingungen während der Weiterbildung familienfreundlicher, insbesondere aber diejenigen für Frauen an ihrem Arbeitsplatz in der Klinik attraktiver gestaltet und über diese Initiativen bereits während der medizinischen Ausbildung Informationen an weibliche Studierende weitergibt.

2. Der von Albrecht diskutierte Wertewandel in der jüngeren Ärztegeneration definiert auf der Basis sozialwissenschaftlicher Untersuchungen unterschiedliche Wertetypen, unter welchen der Typus des nonkonformen Idealisten besonders häufig vorkomme. Dieser zeichne sich durch einen stark ausgeprägten Entfaltungswert, begrenzte Pflicht- und Akzeptanzwerte sowie eine hohe Bereitschaft zum Engagement aus. Man müsse davon ausgehen, dass solche Bewerber klare Strukturen für ihre Weiterbildung aufgezeigt bekommen wollen und Wert auf die Definition mittel- und langfristiger Ziele legen. Wenn Kliniken diesen Ansprüchen nur unvollkommen nachkämen und Ziele nicht eingehalten würden, sei die Bereitschaft gering, einen Weiterbildungsplatz in der Chirurgie anzustreben und die Motivation zur Umorientierung des Berufswunschs in andere Tätigkeitsbereiche nehme zu. Ein im März 2009 abgehaltener Workshop der DGOU zur Realität der Weiterbildung im Fach Orthopädie und Unfallchirurgie unter dem etwas reißerischen Titel: „Operiert Euch doch selbst - Nachwuchsmangel wegen gefühlter Unzufriedenheit?“ gelangt auf der Basis einer kritischen Analyse zu der Feststellung, dass Bürokratie, Zeitdruck, hohe Arbeitsbelastung, diktatorische Vorgesetzte, mangelhafte Anleitung, fehlendes Feedback und eine uneinheitliche, wenig transparente Umsetzung der Weiterbildungsordnung wesentliche Gründe dafür seien, dass sich junge Ärztinnen und Ärzte anderen Berufsfeldern zuwenden und bundesdeutschen Kliniken den Rücken kehren würden [5]. Das Junge Forum der DGOU formuliert in einer Veröffentlichung auf der Basis von Umfrageergebnissen unter Assistenzärzten und -ärztinnen aus orthopädisch-unfallchirurgischen Krankenhäusern eine Reihe von Zielen, die man zur Verbesserung der Rahmenbedingungen in Aus- und Weiterbildung sowie zur Förderung des wissenschaftlichen Nachwuchses unseres Fachgebiets ins Auge fassen muss [9]. Frau Prof. Nüssler betont in einem Aufsatz über den „Attraktivitätsverlust der Chirurgie - wie lässt sich gegensteuern?“, dass sich junge Ärzte nicht mehr nur aus inhaltlichen Gründen für oder gegen ein bestimmtes Fach entscheiden, sondern dass Arbeitsstrukturen gesucht würden, die mit dem bestehenden Gesellschaftsbild kompatibel seien. Dazu gehörten neben der „work-life balance“ das Arbeitsklima sowie die zu erwartende Zufriedenheit mit der eigenen Tätigkeit [6]. Laut Albrecht muss der Wertewandel der neuen Ärztegeneration zur Einrichtung entsprechender Strukturen in der Personalentwicklung und -führung veranlassen. Herr PD Dr. Schröder erklärt in einem Statement zum Auftakt der Kampagne „nur Mut“, dass die deutsche Chirurgie nicht nur von Patienten, sondern auch vom Nachwuchs an ihren Taten gemessen werde. Langfristiges Interesse könne man nur dann wecken, wenn Arbeitsbedingungen und Verdienst stimmen und die Weiterbildung eine verlässliche Struktur und Qualität besitzt.

3. Mängel in der Lehre sind laut Albrecht ein weiterer Grund dafür, dass das Bild der Chirurgie bei Studierenden als wenig attraktiv empfunden würde. Der Unterricht erfolge häufig unter Zeitdruck und durch unerfahrene Dozenten, die nicht über die notwendige Lehrerfahrung verfügten. Die DGOU habe dieses Problem erkannt und daher in 2009 eine Arbeitsgemeinschaft „Lehre“ gegründet. Diese habe sich zur Aufgabe gemacht, Empfehlungen zur Ausbildung von Dozenten zu erarbeiten, Eckpunkte zu einer praktisch orientierten Lehre zu formulieren und eine strukturierte Ausbildung für das „Praktische Jahr“ zu erarbeiten. In den Jahren 2008 und 2009 wurde auf Initiative der Deutschen Gesellschaft für Chirurgie unter Leitung von Prof. Brücher in Tübingen die 1. und 2. Billroth-Akademie für Studierende durchgeführt, die unter Einbeziehung aller chirurgischen Fächer der Eberhard Karls Universität theoretische Kenntnisse und vor allem praktische Inhalte vermittelten. Die unfallchirurgisch-orthopädische Summer School der DGOU im September 2009 an der Universität Freiburg, geleitet von Prof. Südkamp unter Mitwirkung des Jungen Forums, widmete sich ebenfalls dem Thema der Attraktivitätssteigerung des gemeinsamen Faches und erfreute sich einer regen Beteiligung durch interessierte Studierende. Im Rahmen des DKOU 2009 in Berlin startete unter Leitung von Frau PD Dr. Seifert die Kampagne „Stipendium für Studierende“ der DGOU, bei welcher aus einer großen Zahl von Bewerbern 100 Teilnehmer ausgesucht wurden, die mit finanzieller Unterstützung durch die Fachgesellschaften die Möglichkeit erhielten, in Begleitung sog. „Bärenführer“ (etablierte Unfallchirurgen und Orthopäden) Einblicke in Wissenschaft und Forschung, Alltag und Praxis sowie die Berufspolitik unseres Faches zu gewinnen [7].

Dies sind einige der Initiativen, die mit Sicherheit dazu geeignet sind, die Attraktivität der chirurgischen Fächer zu erhöhen und somit dem Nachwuchsmangel in der Chirurgie zu begegnen. In Verbindung mit einer Qualitätsverbesserung der chirurgischen Lehre ist die Fortsetzung solcher Aktionen wesentliche Voraussetzung dafür, dass sich in Zukunft eine zunehmende Zahl von Absolventen des Medizinstudiums für die Weiterbildung in einem chirurgischen Fach, in Sonderheit und gerade auch mit der Ausrichtung auf Orthopädie und Unfallchirurgie entscheidet.

„Die Jugend ist mit Achtung zu betrachten; denn wie kann man wissen, ob die Künftigen nicht besser als die Heutigen sein werden.“ (Konfuzius)

\section{K. Weise, Tübingen}

F.U. Niethard, Aachen

\section{Literatur}

1 Albrecht D. Nachwuchsmangel in der Chirurgie - was tun? Mitteilungen der Deutschen Gesellschaft für Chirurgie 2010; 39: 33-34

2 Ansorg J, Krones C, Schröder Wet al. Auftakt durch Kampagne: Nur Mut! Der Chirurg BDC 2008; 2: 52-53

3 Bauer $H$. Chirurgie - neue Impulse für die Weiterbildung. Der Chirurg BDC 2009; 12: 647-652

4 Münzberg M, Dragowsky K, Depeweg D. „Profil zeigen“ - Time for Change. Z Orthop Unfall 2009; 147: 653-655

5 Niethard FU, Siebert H, Tempka A. „Operiert euch doch selbst“ - Nachwuchsmangel wegen gefühlter Unzufriedenheit? DGU Mitteilungen und Nachrichten 2009; 60: 33-34

6 Nüssler NC. Attraktivitätsverlust der Chirurgie - Wie lässt sich gegensteuern? Der Chirurg BDC 2009; 3: 132-133

7 Seifert J. Die Chance wahrnehmen: Nachwuchsmangel fordert Umdenken in der Unfallchirurgie. DGU Mitteilungen und Nachrichten 2009; 60: $56-58$

8 Weise K, Niethard FU. Das Junge Forum der DGOU - Über die Wünsche und Vorschläge des Nachwuchses in Unfallchirurgie und Orthopädie. Z Orthop Unfall 2009; 147: 15-16

9 Witte D, Moradi B. Wie sieht es aus in deutschen Krankenhäusern? Z Orthop Unfall 2009; 147: 403-404 